\title{
More discussions needed about resuscitation? DNAR orders in a district general hospital
}

\author{
Authors: Mike Pynn, Endaf Morgan, Bethan Gibson and Amit Benjamin
}

\section{Aims}

Following a recent landmark trial and current guidelines, this study examined general medical practice regarding the institution of do not attempt resuscitation (DNAR) orders in a district general hospital, exploring timeliness, appropriateness of completion and the proportion of relevant patients in whom they were considered.

\section{Methods}

A retrospective case note review of 102 medical inpatients was conducted over a 4 -week period and basic demographics, admitting/current diagnoses, comorbidities, functional level and length of stay were recorded. Existing DNAR orders were audited in accordance with national guidelines. An anonymised database of this information was reviewed by both an independent blinded consultant intensivist and a physician, who were asked to comment on the benefits of cardiopulmonary resuscitation (CPR) in the event of deterioration.

\section{Results}

29/102 medical inpatients (28.4\%) had a DNAR order, completed at a median of 2 days after admission. Poor premorbid functional level was the only measure associated with DNAR orders ( $\mathrm{p}<0.001) .62 .1 \%$ of forms were completed by specialist trainees, while only $10.3 \%$ by consultants. $17.2 \%$ of decisions were made by ward cover and $10.4 \%$ by hospital at night doctors; none of these involved a new diagnosis at the time of completion. In $13 / 29$ patients (46.4\%) with DNAR orders assessed to have capacity, five of the decisions (38.5\%) were not documented as being discussed with them. Next of kin were informed in $75.9 \%$ of cases. 30-day mortality was higher in those with a DNAR order $(41.4 \%$ versus $9.6 \%, \mathrm{p}<0.001)$. Independent consultant review concurred with $93 \%$ of existing orders. They concluded a further 27 patients may not benefit from CPR. None of these additional patients' notes had documentation about discussions relating to the burdens of CPR, despite a median length of stay of 10 days. The concordance rate between the consultant's decisions was high (72.5\%).

\section{Conclusions}

DNAR orders are still not discussed with a significant proportion of inpatients, a population with a high mortality. Despite a move towards consultant-led care, DNAR decisions are made by doctors in training in the majority of cases and a significant number by on-call teams. Independent consultant review showed these decisions are reproducible and demonstrated that $54.9 \%$ of medical inpatients may not benefit from CPR, double the number who had an existing order in place. 